\title{
Psychophysical characterisation of early functional loss in glaucoma and ocular hypertension
}

\section{E A Ansari, J E Morgan, R J Snowden}

Br J Ophthalmol 2002;86:1131-1135

See end of article for authors' affiliations

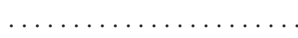

Correspondence to: James E Morgan, Department of Ophthalmology, University Hospital of Wales, Heath Park, Cardiff CF4 4XN UK;

morganje3@cardiff.ac.uk

Accepted for publication 15 May 2002

\begin{abstract}
Aim: The psychophysical evaluation of selective cell loss in early glaucoma and ocular hypertension. Methods: Contrast sensitivity was measured for the detection of luminance modulated gratings at a range of spatial $(0.5,2,8 \mathrm{c} / \mathrm{deg})$ and temporal $(0,16 \mathrm{~Hz})$ frequency combinations in three groups of age matched patients (primary open angle glaucoma, ocular hypertension, normal controls; $n=16$ ). Stimuli of $5^{\circ}$ were presented foveally and at $15^{\circ}$ along the nasal horizontal meridian under photopic conditions.

Results: Fovea: Compared to the normal group, the thresholds for the glaucoma patients were significantly elevated at all spatial and temporal frequencies $(p<0.0001)$, but this reduction was not significantly different at any particular spatial or temporal frequency $(p>0.1)$. There was no difference in contrast sensitivity between the normals and OHTs ( $p>0.10)$. Periphery: The thresholds of the glaucoma patients were elevated compared to the normal controls $(p<0.01)$. The loss of sensitivity was slightly greater at the higher spatial frequencies for both stationary and flickering patterns but this did not reach significance $(p=0.09)$. The contrast sensitivity in normal and OHT groups was not significantly different ( $p>0.10)$.

Conclusions: In early glaucoma, the reduction in contrast sensitivity to stimuli which isolate the magnocellular pathway $(0.5 \mathrm{c} / \mathrm{deg}, 16 \mathrm{~Hz})$ was not significantly different compared with the reduction in contrast sensitivity to stimuli that isolate the parvocellular pathway. These findings are not consistent with the hypothesis that the magnocellular pathway is selectively damaged in early glaucoma.
\end{abstract}

$\mathrm{H}$ istological studies in the early stages of both human and experimental glaucoma have shown a selective reduction in the proportion of larger retinal ganglion cells. ${ }^{1-3}$ Since magnocellular cells tend to be larger at every eccentricity, it is reasonable to conclude that this would translate into a selective loss of magnocellular function. The important clinical implication is that the selective evaluation of magnocellular function should facilitate the earlier detection of ganglion cell loss in glaucoma, possibly before the development of field defects as detected by available clinical perimetric tests.

Although a number of studies have selectively tested either magnocellular or parvocellular pathways in early glaucoma, ${ }^{4-12}$ selective damage to the magnocellular pathway has not been a robust and consistent finding. ${ }^{13-15}$ When magnocellular (M) and parvocellular (P) functions are assessed using a battery of tests a selective deficit in magnocellular function does not appear to be a consistent feature of early glaucoma. ${ }^{16}$

The discrepancy between the anatomical data and psychophysical data questions the anatomical basis for selective cell death. A key point in extrapolating changes in cell size distributions to changes in the proportions of particular classes of cells has been the assumption that the normal relation between cell size and cell type is preserved in disease. Recent anatomical data in the experimental primate glaucoma model suggest that this may not be the case since parvocellular and magnocellular retinal ganglion cells shrink before cell death $^{17}{ }^{18}$; the magnitude of shrinkage was similar in both cell types and would be sufficient to account for the apparent selective loss of larger retinal ganglion cells. ${ }^{19}$

The conflicting results from psychophysical studies may stem from their use as diagnostic tools rather than in the detailed evaluation of magnocellular and parvocellular function. The evaluation of the relative damage to the two pathways should be done using tests with minimal bias in contrast with the rationale for the development of selective tests of retinal ganglion cell function for the diagnosis of glaucomatous damage. Since the characterisation of the magnocellular and parvocellular pathways in healthy eyes is conventionally performed using temporal and spatially modulated sine wave gratings as test stimuli, ${ }^{20}$ these can be applied to the assessment of these pathways in glaucoma patients. The gratings should be presented over a range of stimulus contrasts and spatial frequencies to span the dynamic range of the two pathways since the analysis of selected parts of the contrast sensitivity function for either pathway is unlikely to provide a full characterisation of the damage to any particular pathway. For example, a recent detailed study of the differences in the cut-off spatial frequencies at reduced sensitivities showed a greater change for the magnocellular pathway, ${ }^{21}$ which is not necessarily a result of selective damage to the magnocellular pathway but simply a reflection of the expected difference in the contrast sensitivity for the two pathways at differing sensitivities. ${ }^{20}$

In view of the recent anatomical findings and the need to clarify the results of previous psychophysical studies, we performed psychophysical tests in normal and glaucoma patients to determine relative damage to the magnocellular and parvocellular pathways. The stimuli were designed to isolate as much as possible magnocellular and parvocellular pathways. Gratings were presented at a range of spatial and temporal frequencies to construct full contrast sensitivity functions for our different patient groups and in such a way to minimise any bias in testing. We hypothesise that a selective loss of magnocellular function would be manifest as a relatively greater reduction of sensitivity to gratings with higher temporal and lower spatial frequencies.

\section{METHODS}

Informed consent was obtained from all subjects in accordance with the tenets of the Declaration of Helsinki and the 
Table 1 Patient details

\begin{tabular}{|c|c|c|c|c|}
\hline Group & Male:female & Age range (years) & Mean age (years) & SD \\
\hline Normal $(n=16)$ & $9: 7$ & $41-74$ & 61.1 & 9 \\
\hline OAG $(n=16)$ & $6: 10$ & $59-74$ & 68.1 & 5.1 \\
\hline $\mathrm{OHT}(n=16)$ & $6: 10$ & $40-75$ & 59.3 & 13.5 \\
\hline
\end{tabular}

guidelines of the Bro Taf ethics committee. Primary open angle glaucoma (POAG) and ocular hypertensive (OHT) patients were recruited from the university hospital glaucoma clinic and normal controls from the university optometry clinic.

Contrast sensitivity (the reciprocal of contrast threshold) was measured for the detection of luminance modulated sinusoidal gratings with a variety of spatiotemporal frequency combinations presented in either the central or peripheral visual field.

\section{Patients}

Sixteen patients with early to moderate glaucomatous visual field loss, 16 age matched normal control subjects, and 16 age matched patients with OHT were evaluated. One eye, which fulfilled the inclusion criteria, was tested in each patient. In the glaucoma group, the mean deviation on automated Humphrey field analyser perimetry (San Leandro, CA, USA) using the central 24-2 threshold test ranged between $-0.70 \mathrm{~dB}$ to $-6.4 \mathrm{~dB}$ with corresponding thinning of the neuroretinal rim by established criteria. ${ }^{22}$

All patients had a corrected Snellen visual acuity of at least 20/30 achieved with less than plus or minus 4 dioptres (D) spherical equivalent and less than $2 \mathrm{D}$ of astigmatism. The refractive error was corrected with reference to published data. ${ }^{23}$ There was no history of ocular or neurological disease, with the exception of OAG in the experimental group. In addition, patients were included in the OHT group if the intraocular pressure (IOP) exceeded $21 \mathrm{~mm} \mathrm{Hg}$ on two or more occasions by Goldmann contact tonometry, and two or more normal Humphrey field analyser 24-2 examinations. All subjects had comprehensive eye examinations to document anterior segment, open iridocorneal angles (Shaeffer grade III-IV), posterior pole, macular region, and optic nerve head. Subjects with POAG had been diagnosed at least one year previously and intraocular pressures (IOPs) were less than $21 \mathrm{~mm}$ $\mathrm{Hg}$ following treatment with carteolol and/or latanoprost drops.

No patients were taking pilocarpine. All fields were deemed reliable if false positive, false negative, and fixation loss data were $<25 \%$. $^{14}$

\section{Stimuli}

Stimuli were produced by a Cambridge Research Systems VSG waveform generator housed in a PC computer. They were displayed on a CRT (Joyce Electronics, Cambridge, UK). The display had a white phosphor (P4), a mean luminance of 120 $\mathrm{cd} / \mathrm{m}^{2}$ and was refreshed at $100 \mathrm{~Hz}$. The output voltage from the VSG was gamma corrected to ensure linearity up to $90 \%$ contrast. An adjustable target was presented to aid fixation.

All stimuli had a sinusoidal luminance profile along the horizontal axis and were presented within a window of 5 degrees, the edges of which were attenuated using a cosine ramp subtending 0.75 degrees. The rest of the screen ( 16 by 16 degrees) was kept at the mean screen luminance. Each grating was presented for $300 \mathrm{~ms}$ at its full contrast and was linearly ramped on and off over a period of $100 \mathrm{~ms}$ to ensure that the stationary stimulus did not have any high temporal frequency artefacts. All stimulus contrasts are Michaelson contrasts:

$\left.\left(\operatorname{Lum}_{\max }-\mathrm{Lum}_{\min }\right) / \mathrm{Lum}_{\max }+\mathrm{Lu}_{\min }\right)$

and are presented in $\mathrm{dB}$ relative to a contrast of 1 . A contrast of 0.1 is therefore equivalent to $20 \mathrm{~dB}$ of attenuation.

\section{Procedure}

The display was viewed monocularly and the contralateral eye was occluded with an eye patch. The subject's head was stabilised using a headrest at a viewing distance of $57 \mathrm{~cm}$. All patients were allowed enough time to adapt to the ambient testing conditions in the laboratory. All measurements were completed in one or two sessions, each lasting 20-30 minutes.

Experiments were performed with central or eccentric fixation. For foveal viewing three spatial frequencies $(0.5,2$, and 8 $\mathrm{c} / \mathrm{deg}$ ) were tested at each of two temporal frequencies ( 0 and $16 \mathrm{~Hz}$ ). These combinations were chosen to ensure that the lowest spatial and high temporal frequency would best detect lesions of the magnocellular pathway. ${ }^{24}$

For evaluation of the peripheral visual field, gratings were presented 15 degrees nasal to fixation on the horizontal meridian, which corresponded to a region of visual field defect on Humphrey perimetry in all patients. On the pattern deviation plot, fewer than $25 \%$ of points were depressed below the $5 \%$ level and fewer than 10 points are depressed below the $1 \%$ level. No point in the central 5 degrees had a sensitivity of less than $15 \mathrm{~dB}$. The same combinations of spatial and temporal frequencies were tested with the exception of the $8 \mathrm{c} / \mathrm{deg}$ grating, which was omitted because of the low resolution acuity at this eccentricity. ${ }^{25}$

At each location, contrast sensitivity was determined using a binary choice procedure in which the grating presentation was randomly assigned to one of two presentation intervals. The contrast of the grating was adjusted via an adaptive staircase procedure (QUEST), ${ }^{26}$ in which contrast was increased following incorrect responses and decreased following correct responses, converging to a performance level of $81.6 \%$ correct. The initial test contrast was $10 \mathrm{~dB}$ above threshold based upon pilot data.

Each trial consisted of two intervals separated by $250 \mathrm{~ms}$. In one interval (randomly chosen) a grating was presented while in the other the screen remained blank. The procedure continued for 32 trials. For each set of trials only a single combination of spatial and temporal frequency was tested. The ordering of the various spatial and temporal frequencies was randomised from observer to observer. All tests on foveal vision were completed before any tests of eccentric vision. Statistical analysis for normally distributed data, unless otherwise stated, was by a two way analysis of variance (ANOVA)

\section{RESULTS}

The POAG and OHT groups both comprised 10 females and six males. The normal (control) group comprised seven females and nine males (Table 1). The mean age for each group (means (SD)) were POAG 68.1 (5.1), OHT 59.3 (13.5), controls 61.1 (9.00) and did not differ significantly $(p=0.2)$. In the glaucoma group the mean deviation on automated Humphrey field analyser perimetry (San Leandro, CA, USA) using the central 24-2 threshold test was 2.3 (1.9), range $-0.70 \mathrm{~dB}$ to $-6.4 \mathrm{~dB}$.

\section{Foveal presentation}

Contrast sensitivities for stationary and flickering gratings are shown in Figure 1. For all groups the results are consistent with previous work in showing bandpass characteristics for 

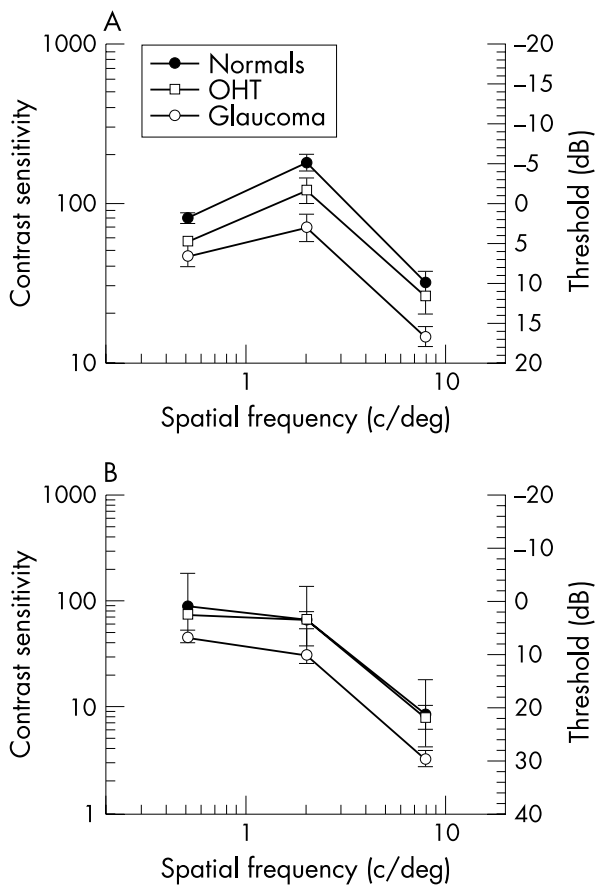

Figure 1 (A) Contrast sensitivity function for the three patient groups - foveal viewing, stationary stimuli $(\mathrm{O} \mathrm{Hz})$. (B) Contrast sensitivity function for the three patient groups-foveal viewing, flickering stimuli $(16 \mathrm{~Hz})$. Error bar (SEM).

low temporal frequencies and low pass functions for high temporal frequencies. ${ }^{20} 27$

Compared to the normal group, the thresholds for the glaucoma patients were significantly reduced at all spatial $(p<0.0001)$ and temporal frequencies $(p<0.0001)$, but this reduction was not significantly different at any particular spatial or temporal frequency $(\mathrm{p}>0.1)$.

There was no difference in contrast sensitivity between the normals and OHTs $(p>0.10)$. However, Figure 2 shows that normals and OHTs showed a greater difference with stationary compared with flickering gratings $(p=0.07)$.

\section{Peripheral presentation}

Data for one normal control, three glaucoma patients, and two OHT patients were not included in the analysis since they could not detect the stimulus even at full contrast. Contrast sensitivities for the remaining observers in each group are plotted in Figure 2. The thresholds of the glaucoma patients are reduced compared with the normal controls $(\mathrm{p}<0.01)$. The loss of sensitivity was greater at the higher spatial frequencies for both stationary and flickering patterns but this was not statistically significant $(p=0.09)$. There was no significant reduction in contrast sensitivity between normal and OHT groups $(\mathrm{p}>0.10)$.

Since a selective loss of magnocellular cell function should produce greater loss of sensitivity for low spatial, high temporal frequencies, we compared the loss of sensitivity under this condition $(0.5 \mathrm{c} / \mathrm{deg}, 16 \mathrm{~Hz})$-with gratings least likely to activate magnocellular cells (and most likely to isolate parvocellular cell function) - with spatial and temporal frequencies as of $8 \mathrm{c} / \mathrm{deg}, 0 \mathrm{~Hz}$ for the fovea and $2 \mathrm{c} / \mathrm{deg}, 0 \mathrm{~Hz}$ for the periphery. At the fovea we found a slightly greater loss for the latter condition $(6.88 \mathrm{~dB} ; t$ test $\mathrm{p}<0.01)$ than the former $(5.63$ $\mathrm{dB} ; t$ test $\mathrm{p}<0.01)$. In the periphery, the low spatial, high temporal conditions produced less loss $(4.3 \mathrm{~dB} ; t$ test $\mathrm{p}<0.05)$ than the high spatial low temporal condition $(6.15 \mathrm{~dB}$; $t$ test $\mathrm{p}<0.01$ ).
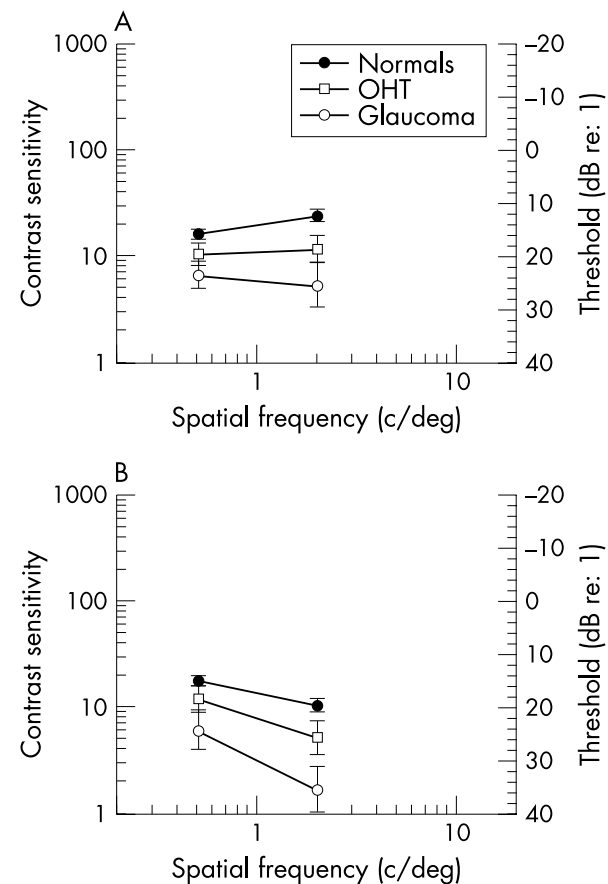

Figure 2 (A) Contrast sensitivity function for the three patient groups-peripheral viewing, stationary stimuli $(\mathrm{O} \mathrm{Hz})$. (B) Contrast sensitivity function for the three patient groups-peripheral viewing, flickering stimuli (16 Hz). Error bar (SEM).

\section{DISCUSSION}

Experimental selective disruption of the magnocellular pathway has revealed reduced sensitivity for low spatial and high temporal frequencies, ${ }^{24}$ whereas lesions of the parvocellular pathway produce deficits across the rest of the spectrum. ${ }^{28}$ If the magnocellular pathway is selectively damaged in glaucoma patients we should expect to see relatively greater reductions in sensitivity compared to normal controls as our stimuli enter this low spatial, high temporal frequency domain.

In the present study we found significant losses of contrast sensitivity in our glaucoma group compared to normals across the range of spatiotemporal frequencies. We were unable to demonstrate a greater loss of sensitivity for either lower spatial or higher temporal, or the combination of low spatial, high temporal frequencies. The one trend that did reach statistical significance was a greater reduction in sensitivity for the glaucoma patients for the detection of higher spatial frequency stimuli presented in the peripheral retina. Sensitivity for OHT patients was midway between POAG and control groups.

There are several important technical limitations with studies that attempt to isolate the magnocellular and parvocellular streams. The correlation of our findings with the anatomical data is complicated since recent studies suggest that the two channels are not clearly segregated. ${ }^{29}$ A subset of parvocellular cells (bistratified RGCs) with large fibre diameter processes short wavelength cone information ${ }^{30}{ }^{31}$ resulting in an overlap of structure and function in which it is very difficult to ascribe function to a particular cell based on its dimensions. Although there is no independent test for M cells, we used spatiotemporal frequency combinations that should provide optimal separation. Furthermore, this approach would minimise the extent to which $\mathrm{P}$ cells mask $\mathrm{M}$ cell deficits.

Our findings differ from those of Anderson and O'Brien, ${ }^{21}$ who employed a similar test paradigm and expressed the degree of relative damage to magnocellular and parvocellular pathways as a ratio of the response to the flickering and stationary stimuli. The ratio was reduced in glaucoma patients 

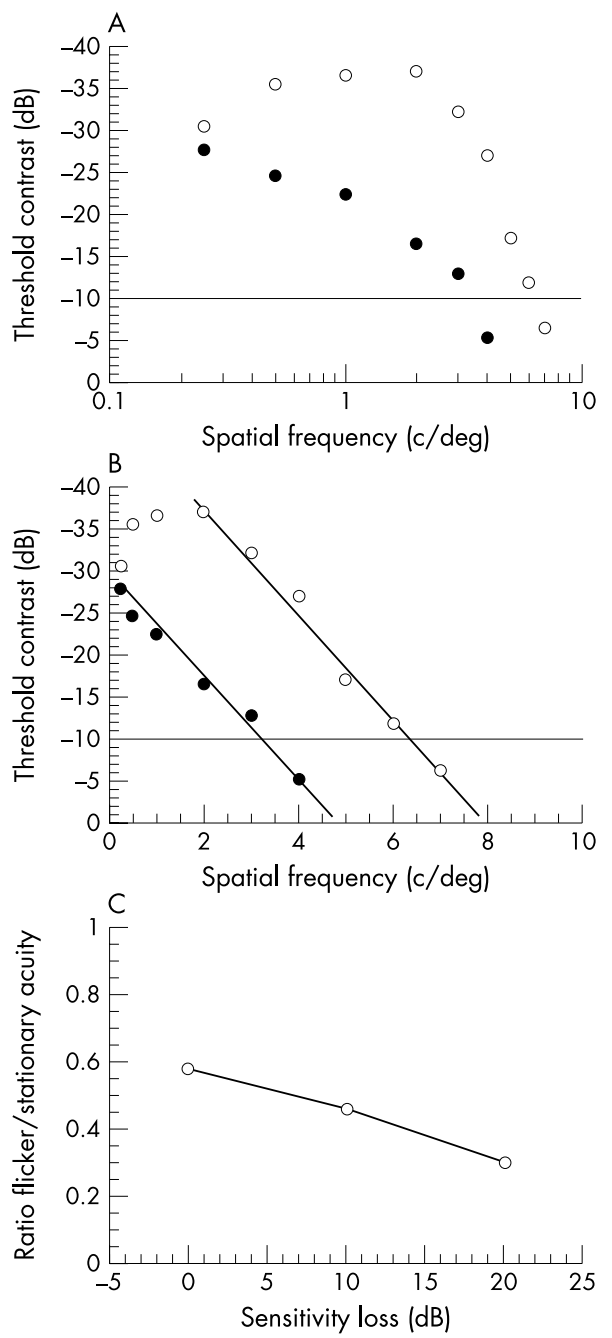

Figure 3 Graphs demonstrating that non-selective loss can account for the change in contrast sensitivity ratios between the $M$ and $P$ cell pathways. Contrast sensitivity functions (CSF) have been plotted for stimuli that are either stationary (open circles) or counterphase flickered (solid circles) observed by a single, representative (non-glaucomatous) observer. (A) shows that with logarithmic axes, the slope for the loss of sensitivity at high spatial frequencies differs for the two stimuli. (B) shows matching slopes for the two stimuli when plotted on linear axes. (C) The change in the ratio of acuity for moving and stationary stimuli arises from the relative shape of the contrast sensitivity functions for these stimuli in the normal eye.

compared to controls, which was interpreted as evidence for selective loss of magnocellular cells in glaucoma. Figure 3 illustrates how their findings are also consistent with non-selective damaged to both magnocellular and parvocellular pathways. Contrast sensitivity functions (CSF) have been plotted for stimuli which are either stationary or counterphase flickered observed by a single, representative (nonglaucomatous) observer. With logarithmic axes, the slope for the loss of sensitivity at high spatial frequencies differs for the two stimuli (Fig 3A) but when replotted on linear axes the slopes are the same (Fig 3B). At maximum contrast, the cut off (resolution) acuity is $8 \mathrm{c} / \mathrm{deg}$ for stationary gratings and 5 c/deg for flickering gratings giving a ratio of flickering to stationary stimuli of $5 / 8=0.6$. The effect of glaucoma on these contrast sensitivity functions can be simulated by measuring the cut-off acuities when the sensitivity is reduced by $10 \mathrm{~dB}$. In this example, the acuity is $6.4 \mathrm{c} / \mathrm{deg}$ for stationary gratings and $3.4 \mathrm{c} / \mathrm{deg}$ for flickering gratings, ratio $=3.4 / 6.4=0.53$. The ratio for the acuity at resolution for the two gratings (Fig 3C) is decreased as the sensitivity is reduced because the sensitiv- ity for flickering gratings is lower at every spatial frequency. The change in ratio is not the result of differential damage to magnocellular pathway but comes from the relative shape of the contrast sensitivity functions for moving and stationary stimuli in the normal eye.

Our findings are consistent with a recent study of photopic and scotopic motion sensitivity in POAG patients showing that moving gratings, optimised for the stimulation of magnocellular pathways provided relatively poor discrimination of normal from glaucomatous eyes. ${ }^{32}$ This study also showed that age was an important confounding factor when studying motion detection thresholds. Since thresholds for motion detection increase with increasing age, this would reduce the value of motion detection tasks for the diagnosis of glaucoma.

Clinical studies in which comparable psychophysical or electrophysiological tests of magnocellular and parvocellular cell function have been used in patients with early glaucoma have also failed to find strong evidence for a selective magnocellular damage. The pattern electroretingram (PERG) and letter acuity tests had the best sensitivity and specificity, but motion detection and flicker contrast performed poorly. ${ }^{16}$ When tests are performed to isolate magnocellular and parvocellular cell function at different retinal locations, defects are found in both systems in early POAG, with worst deficits in the superior visual field. ${ }^{15}$ In addition, there is evidence that red-green colour perception may also be compromised in early glaucoma. ${ }^{334}$

It is possible that the failure to find any selective damage reflects the difficulties associated with isolation of the functional properties of the magnocellular and parvocellular pathways. Partly this is due to the overlapping function of the two pathways since the parvocellular pathway conveys information about the motion of moderate and high spatial frequency targets. ${ }^{2435}$ Clinical tests of magnocellular function-for example, using moving lines or dots may be complicated by the broad Fourier spectra of these stimuli which would stimulate both magnocellular and parvocellular cells. ${ }^{32}$ Further difficulties come from the convergence of the two pathways at the level of the striate cortex. Electrophysiological measures of cortical function may provide better separation of the two pathways. Indeed, pseudorandomly stimulated flash visual evoked potentials (VEP) ${ }^{36}$ suggest a preferential loss of magnocellular cells in early glaucoma with the parvocellular component declining later on in the disease. The degree to which these techniques separate the pathways requires further confirmation but these data suggest that selectivity may have a greater effect at the cortical compared with the thalamic level.

In clinical terms, non-selective RGC loss does not undermine selective testing of those pathways with reduced redundancy. ${ }^{13}$ Thus, frequency doubling perimetry (FDP) is sensitive to defects in an undersampled, non-linear subset of magnocellular cells, the My cells, and has high sensitivity and specificity for the discrimination of normal from glaucomatous eyes. ${ }^{37}{ }^{38}$ Bistratified RGCs comprise $1 \%$ of RGCs in the central retina and mediate the blue-yellow signal. ${ }^{30}$ Reduced redundancy in this population of cells may explain the ability of blue on yellow perimetry to detect visual field loss earlier than conventional perimetry. ${ }^{39}$

Non-selective cell death has implications for our understanding of the events that lead to retinal ganglion cell death. One interpretation of non-selective cell loss is that cell shrinkage occurs in glaucoma to account for the shift in cell soma size distributions in the disease. ${ }^{17}{ }^{18}$ In the primate model of glaucoma, RGC shrinkage is characterised by reduction in size of the dendritic tree, ${ }^{18}$ cell soma, and axon to a similar extent in both magnocellular and parvocellular cells. This is consistent with glaucomatous retinal ganglion cell damage being diffuse and having widespread effects on the cells before cell death. ${ }^{40}$ Since these cells are at risk of cell death they may benefit from measures aimed at cell rescue. How these cells 
might be identified physiologically is unclear. Reduced dendritic arbor could translate into a smaller receptive field ${ }^{41}$ though it would be difficult to predict the behaviour of the remaining dendrites.

In summary, we have not found evidence for selective damage to the magnocellular pathway in early glaucoma. While complete isolation of magnocellular function may not be possible, our data suggest that selective magnocellular damage, if it does occur, is not a significant effect.

\section{ACKNOWLEDGEMENTS}

Supported by the International Glaucoma Association, UK and Pharmacia and Upjohn, UK.

Presented in part at the Association for Research in Vision and Ophthalmology (ARVO), Fort Lauderdale, 2000.

Proprietary interest: nil.

\section{Authors' affiliations}

E A Ansari, J E Morgan, Department of Ophthalmology, University of Wales College of Medicine, Cardiff, UK

R J Snowden, Department of Psychology, Cardiff University, Cardiff, UK

\section{REFERENCES}

1 Glovinsky Y, Quigley HA, Dunkelberger GR. Retinal ganglion cell loss is size dependent in experimental glaucoma. Invest Ophthalmol Vis Sci 1991;32:484-91.

2 Glovinsky Y, Quigley HA, Pease ME. Foveal ganglion cell loss is size dependent in experimental glaucoma. Invest Ophthalmol Vis Sci 1993;34:395-400

3 Quigley HA, Dunkelberger GR, Green R. Chronic human glaucoma causing selectively greater loss of large optic nerve fibres. Ophthalmology 1988;95:357-65.

4 Drum B, Severns M, O'Leary D, et al. Selective loss of pattern discrimination in early glaucoma. Appl Opt 1989; 28: 1135-44.

5 Fitzke FW, Poinooswamy D, Ernst W, et al. Peripheral displacement thresholds in normals, ocular hypertensives and glaucoma. Doc Ophthalmol 1986:49:447-52.

6 Frisen L. High-pass resolution perimetry. Recent developments. In: Heij A, ed. Perimetry update 1988/89. Amsterdam: Kugler and Ghedini, 1989:369-76.

7 Johnson CA, Adams AJ Casson EJ, et al. Blue-on-yellow perimetry can predict the development of glaucomatous visual field loss. Arch Ophthalmol 1993; 111:645-50.

8 Joffe KE, Raymond JE, Crichton A. Motion coherence perimetry in glaucoma and suspected glaucoma. Vis Res 1997; 37:955-64.

9 Sample PA, Madrid ME, Weinreb RN. Evidence for a variety of functional defects in glaucoma suspect eyes. J Glaucoma 1994;3(suppl 1):S5-18.

10 Sample PA, Weinreb RN. Progressive colour visual field loss in glaucoma. Invest Ophthalmol Vis Sci 1992;33:2068-71.

11 Silverman SE, Trick GL, Hart WM. Motion perception is abnormal in primary open angle glaucoma and ocular hypertension. Invest Ophthalmol Vis Sci 1990; 31:722-9.

12 Tyler CW. Specific defects of flicker sensitivity in glaucoma and ocular hypertension. Invest Ophthalmol Vis Sci 1981;100:135-46.

13 Johnson CA. Selective versus nonselective losses in glaucoma. J Glaucoma 1994;3(suppl 1):S32-44.

14 Sample PA, Bosworth CF, Weinreb RN. Short-wavelength automated perimetry and motion automated perimetry in patients with glaucoma. Arch Ophthalmol 1997;115:1129-33.
15 Swindale NV, Fendick M, Drance SM, et al. Contrast sensitivity for flickering and static letters and visual acuity at isoluminance in glaucoma. J Glaucoma 1996;5:156-69.

16 Graham SL, Drance SM, Chauhan BC, et al. Comparison of psychophysical and electrophysiological testing in early glaucoma. Invest Ophthalmol Vis Sci 1996;37:2651-62.

17 Morgan JE, Uchida H, Caprioli J. Retinal ganglion cell death in experimental glaucoma. Br J Ophthalmol 2000;84:303-10.

18 Weber AJ, Kaufman P, Hubbard WC. Morphology of single ganglion cells in the glaucomatous primate retina. Invest Ophthalmol Vis Sci 1998; 39:2304-20.

19 Morgan JE. Selective cell loss in glaucoma: does it really occur? $\mathrm{Br} J$ Ophthalmol 1994;78:875-80.

20 Robson JG. Spatial and temporal contrast sensitivity functions of the visual system. J Opt Soc Am 1966; 56:1141-2.

21 Anderson RS, O'Brien C. Psychophysical evidence for a selective loss of $M$ ganglion cells in glaucoma. Vis Res 1997;37:1079-83.

22 Jonas JB, Fernandez MC, Sturmer J. Pattern of glaucomatous neuroretinal rim loss. Ophthlamology 1993;100:63-8.

23 Millodot $\mathbf{M}$, Millodot S. Presbyopia correction and the accommodation in reserve. Ophthal Physiol Opt 1989;9:126-32

24 Merigan WH, Maunsell JHR. Macaque vision after magnocellular lateral geniculate lesions. Visual Neurosci 1990;5:347-52

25 Pointer JS, Hess RF. The contrast sensitivity gradient across the human visual field: with emphasis on the low spatial frequency range. Vis Res 1989;29:1133-51.

26 Watson AB, Peli DG. QUEST: a Bayesian adaptive psychometric method. Perception and Psychophysics 1983:33:113-20.

27 Atkin A, Bodis-Wollner I, Wolkstein M, et al. Abnormalities of central contrast sensitivity in glaucoma. Am J Ophthalmol 1979;88:205-2.

28 Merigan WH, Katz LM, Maunsell JHR. The effects of parvocellular lateral geniculate lesions on the acuity and contrast sensitivity of macaque monkeys. J Neurosci 1991;11:994-1001.

29 Merigan WH, Maunsell JHR. How parallel are the primate visual pathways? Ann Rev Neurosci 1993;16:369-402.

30 Dacey DM, Lee BB. The blue-ON opponent pathway in primate retina originates from a distinct bistratified ganglion cell type. Nature 1994;367:731-5

31 DeMonastario FM. Asymmetry of on- and off-pathways of blue-sensitive cones of the retina of macaque. Brain Res 1979;166:39-48.

32 Willis A, Anderson SJ. Effects of glaucoma and aging on photopic and scotopic motion perception. Invest Ophthalmol Vis Sci 2000;41:325-35

33 Greenstein VC, Halevy D, Zaidi Q, et al. Chromatic and luminance system deficits in glaucoma. Vis Res 1996;36:621-9.

34 Porciatti V, Di Bartolo E, Nardi MM, et al. Responses to chromatic and luminance contrast in glaucoma: a psychophysical and electrophysiological study. Vis Res 1997;37:1975-87.

35 Galvin SJ, Williams DR, Coletta NJ. The spatial grain of motion perception in human peripheral vision. Vis Res 1996;36:2283-95.

36 Klistorner Al, Graham SL. Early magnocellular loss in glaucoma demonstrated using the pseudorandomly stimulated flash visual evoked potential. J Glaucoma 1999;8:140-8.

37 Johnson CA, Samuels SJ. Screening for glaucomatous field loss with frequency-doubling perimetry. Invest Ophthalmol Vis Sci 1997; 38:413-425

38 Quigley HA. Identification of glaucoma-related visual field abnormality with the screening protocol of frequency doubling technology. Am J Ophthalmol 1998;125:819-29.

39 Casson EJ, Johnson CA, Shapiro LR. Longitudinal comparison of temporal-modulation perimetry with white-on-white and blue-on-yellow perimetry in ocular hypertension and early glaucoma. J Opt Soc Am A $1993 ; 10: 1792-806$

40 Henson DB, Artes PH, Chauhan BC. Diffuse loss of sensitivity in early glaucoma. Invest Ophthalmol Vis Sci 1999;40:3147-51

41 Takao M, Miyoshi T, Watanabe M, et al. Receptive field properties of axotomized retinal ganglion cells of adult cats. [ARVO Abstract.] Invest Ophthalmol Vis Sci 2000;41:S938 (abstract no 4988). 